\title{
NEW IDENTITIES INVOLVING SUMS OF THE TAILS RELATED TO REAL QUADRATIC FIELDS
}

\author{
KATHRIN BRINGMANN AND BEN KANE
}

To George Andrews, who has been a great inspiration, on the occasion of his 70th birthday

\begin{abstract}
In previous work, the authors discovered new examples of $q$-hypergeometric series related to the arithmetic of $\mathbb{Q}(\sqrt{2})$ and $\mathbb{Q}(\sqrt{3})$. Building on this work, we construct in this paper sum of the tails identities for which some which some of these functions occur as error terms. As an application, we obtain formulas for the generating function of a certain zeta functions for real quadratic fields at negative integers.
\end{abstract}

\section{INTRODUCTION}

Andrews, Dyson and Hickerson [2] investigated the function

$$
\sigma(q):=1+\sum_{n \geq 1} \frac{q^{\frac{n(n+1)}{2}}}{(-q)_{n}}=1+q-q^{2}+2 q^{3}+\cdots+4 q^{45}+\ldots
$$

which first appeard in Ramanujan's lost notebook [7]. We denote as usual $(a)_{n}:=(a ; q)_{n}:=$ $\prod_{m=0}^{n-1}\left(1-a q^{m}\right)$. By showing that the Fourier coefficients of $\sigma(q)$ are connected to the arithmetic of $\mathbb{Q}(\sqrt{6})$, the authors of $[2]$ were able to prove that $\sigma$ is lacunary, i.e. its coefficents are almost always zero, and yet attains every integer infinitely many times.

Since Andrews, Dyson and Hickerson's investigation, the function $\sigma(q)$ has shown up in a variety of settings and is related to the theory of automorphic forms in many interesting ways, as described in [4]. Here we consider one such type of result involving sum of tails identities. Such identities were first studied by Zagier [8] in his work on Vassiliev invariants, who showed that

$$
\sum_{n \geq 0}\left((q)_{\infty}-(q)_{n}\right)=(q)_{\infty} D_{1}(q)+E_{1}(q)
$$

where

$$
D_{1}(q):=-\frac{1}{2}+\sum_{n \geq 1} d(n) q^{n}, \quad E_{1}(q):=\frac{1}{2} \sum_{n \geq 1}\left(\frac{12}{n}\right) n q^{\frac{n^{2}-1}{24}} .
$$

Here $d(n)$ denotes the number of divisors of $n$ and $\left(\frac{\bullet}{n}\right)$ is the Kronecker symbol. In [3], Andrews, Jimenez-Urroz, and Ono obtained a number of related identites, which have the form

$$
\sum_{n \geq 0}\left(F(q)-F_{n}(q)\right)=F(q) D(q)+E(q)
$$

where $F$ is a (modular) infinite product, $F_{n} \rightarrow F$, and $D$ is a divisor function. Since the coefficients of $E$ grow much slower than those of $F D$, the function $E$ may be considered as an

2000 Mathematics Subject Classification. 11P81, 11E16, 05A17.

Key words and phrases. q-hypergeometric series, sum of tails, modular form, real quadratic fields.

The first author was partially supported by NSF grant DMS-0757907. 
"error series". Of particular interest are special cases of (1.1) in which the "error series" is $\sigma(q)$, for example the following formula which can be found in Ramanujan's lost notebook

$$
\sum_{n \geq 0}\left((-q)_{\infty}-(-q)_{n}\right)=(-q)_{\infty} D_{1}(q)+\frac{1}{2} \sigma(q) .
$$

In [4], the authors discovered 8 additional examples of $q$-hypergeometric series related to the arithmetic of $\mathbb{Q}(\sqrt{2})$ and $\mathbb{Q}(\sqrt{3})$ including the functions

$$
f(q):=\sum_{n \geq 0} \frac{(q)_{2 n}}{(-q)_{2 n+1}} q^{n}, \quad h(q):=\sum_{n \geq 0} \frac{(q)_{2 n+1}}{(-q)_{2 n+2}} q^{n+1} .
$$

Using the theory of Bailey pairs, it was shown in [4] that

$$
\begin{aligned}
q f\left(q^{2}\right) & =\sum_{\mathbf{a} \subset O_{K}}\left(\frac{-4}{\mathcal{N}(\mathbf{a})}\right) q^{\mathcal{N}(\mathbf{a})}, \\
h(q) & =-\sum_{\mathbf{a} \subset O_{K}}(-1)^{\mathcal{N}(\mathbf{a})} q^{\mathcal{N}(\mathbf{a})}
\end{aligned}
$$

where $K:=\mathbb{Q}(\sqrt{2}), O_{K}$ is the ring of integers of $K$, the sum runs over all ideals of $O_{K}$, and $\mathcal{N}$ denotes the norm of an ideal. As in the case of $\sigma$, the identities in (1.3) and (1.4) imply arithmetic information about the coefficients of $f$ and $h$ including lacunary behavior.

In this note we consider sums of tails identities resembling (1.2) which involve the functions $f$ and $h$ as "error series". To state our results, for $i \in\{0,1\}$ we define

$$
\theta_{i}(q):=\sum_{n \in \mathbb{Z}} q^{\frac{1}{2}(2 n+i)^{2}}
$$

and, denoting as usual the $q$-binomial coefficients by $\left[\begin{array}{l}A \\ B\end{array}\right]:=\frac{(q)_{A}}{(q)_{B}(q)_{A-B}}$, we let its "finite companion" be given by

$$
\theta_{i, n}(q):=(q)_{n} \sum_{j \in \mathbb{Z}}\left[\begin{array}{c}
2 n+1 \\
n-2 j
\end{array}\right] q^{\frac{1}{2}(2 j+i)^{2}} .
$$

We note that $\lim _{n \rightarrow \infty} \theta_{i, n}(q)=\theta_{i}(q)$ and that the theta series $\theta_{i}\left(q^{2}\right)$ are modular forms which can be expressed as an infinite product, namely they can be written $\theta_{0}\left(q^{\frac{1}{2}}\right)=\frac{\left(q^{2} ; q^{2}\right)_{\infty}^{5}}{(q)_{\infty}^{2}\left(q^{4} ; q^{4}\right)_{\infty}^{2}}$ and $\theta_{1}\left(q^{2}\right)=2 q \frac{\left(q^{16} ; q^{16}\right)_{\infty}^{2}}{\left(q^{8} ; q^{8}\right)_{\infty}}$. Moreover, we define

$$
D_{2}(q):=\sum_{n=1}^{\infty} d_{o}(n) q^{n},
$$

where $d_{0}(n)$ counts the number of odd divisors of $n$.

Theorem 1.1. We have the sum of tails identities

$$
\begin{aligned}
\sum_{n \geq 0}\left(\frac{\theta_{0}(q)}{(q)_{\infty}\left(q ; q^{2}\right)_{\infty}}-\frac{\theta_{0, n}(q)}{(q)_{n}\left(q ; q^{2}\right)_{n+1}}\right) & =2 \frac{\theta_{0}(q)}{(q)_{\infty}\left(q ; q^{2}\right)_{\infty}} D_{2}(q)-h(q), \\
\sum_{n \geq 0}\left(\frac{\theta_{1}(q)}{(q)_{\infty}\left(q ; q^{2}\right)_{\infty}}-\frac{\theta_{1, n}(q)}{(q)_{n}\left(q ; q^{2}\right)_{n+1}}\right) & =2 \frac{\theta_{1}(q)}{(q)_{\infty}\left(q ; q^{2}\right)_{\infty}} D_{2}(q)+q^{\frac{1}{2}} f(q) .
\end{aligned}
$$


As an application, we next consider formulas for the generating function of the following zeta functions for real quadratic fields at negative integers, paralleling the results obtained in [8] and [3]. For this define the usual Hecke $L$-function $\zeta_{K}(s)$ for $K$ and a twisted zeta-function $\widetilde{\zeta_{K}}(s)$ by

$$
\zeta_{K}(s):=\sum_{\mathbf{a} \subset O_{K}} \mathcal{N}(\mathbf{a})^{-s}, \quad \widetilde{\zeta_{K}}(s):=\sum_{\mathbf{a} \subset O_{K}}\left(\frac{-4}{\mathcal{N}(\mathbf{a})}\right) \mathcal{N}(\mathbf{a})^{-s} .
$$

Theorem 1.1 will give us the following formulas from which $\zeta_{K}(s)$ and $\widetilde{\zeta_{K}}(s)$ may be calculated at negative integers.

Theorem 1.2. As a power series in $t$, we have the equations

$$
\begin{aligned}
-\sum_{n \geq 0} \frac{\theta_{0, n}\left(-e^{-t}\right)}{\left(-e^{-t} ;-e^{-t}\right)_{n}\left(-e^{-t} ; e^{-2 t}\right)_{n+1}} & =\sum_{n \geq 1}(-1)^{n} \zeta_{K}(-n) \frac{t^{n}}{n !}, \\
\sum_{n \geq 0} \frac{\theta_{1, n}\left(-e^{-t}\right)}{\left(-e^{-t} ;-e^{-t}\right)_{n}\left(-e^{-t} ; e^{-2 t}\right)_{n+1}} & =\sum_{n \geq 1}\left(-\frac{1}{2}\right)^{n} \widetilde{\zeta_{K}}(-n) \frac{t^{n}}{n !} .
\end{aligned}
$$

We note that similarly we could also consider the limiting behavior at other odds roots of unity.

This paper is organized as follows. In Section 2, we first introduce an auxilliary function with an extra parameter, which is in special cases related to $f$ and $h$, and thus also to the arithmetic of $K$. This auxiliary function also has some nice combinatorial meaning. We then show that this function is the "error series" in a sum of tails identity. In Section 3, we use the results from Section 2 to prove Theorem 1.1. We conclude section 3 with another sums of tails result involving $h$, but where $h(q)$ is not the "error series." Finally, we will establish the formulas for $\zeta_{K}(s)$ and $\widetilde{\zeta_{K}}(s)$ at negative integers in Section 4.

\section{ACKNOWLEDGEMENTS}

The authors thank George Andrews and Jeremy Lovejoy for fruitful conversations. Moreover, they thank Ken Ono for helpful comments on an earlier version of the paper.

\section{An Auxilliary FunCtion}

We will first investigate the properties of an auxilliary function which we will then bootstrap to obtain the main results for $f$ and $h$. To this end, we define

$$
g_{z}(q):=\sum_{n \geq 0} \frac{(q)_{n}}{(-q)_{n+1}} z^{-n} q^{\frac{n}{2}} .
$$

We will see in equation (2.6) that $z^{-1} q g_{z}\left(q^{2}\right)$ is the generating function for a natural combinatorial object. For this recall that an overpartition of the integer $n$ is a partition, where the last occurence of each part may be overlined. Denote by $P$ the set of overpartitions into odd parts where the largest part must be overlined. If we denote the largest part by $L(\Lambda)$ and the number of parts by $M(\Lambda)$, then the coefficient of $z^{-m} q^{n}$ in $z^{-1} q g_{z}\left(q^{2}\right)$ is the number of overpartitions $\Lambda \in P$ of $n$ with $M(\Lambda)=m$, weighted by $\left(\frac{-4}{L(\Lambda)}\right)$.

We will particularly be interested in the two specializations $z= \pm 1$. For the specialization $z=1$, the $n$-th Fourier coefficient of $q g_{+1}\left(q^{2}\right)$ equals

$$
\#\{\Lambda \in P: L(\Lambda) \equiv 1 \quad(\bmod 4)\}-\#\{\Lambda \in P: L(\Lambda) \equiv 3 \quad(\bmod 4)\},
$$


while the $n$-th coefficient of $q g_{-1}\left(q^{2}\right)$ equals

$$
\#\{\Lambda \in P: L(\Lambda) \equiv 2 M(\Lambda)+1 \quad(\bmod 4)\}-\#\{\Lambda \in P: L(\Lambda) \not \equiv 2 M(\Lambda)+1 \quad(\bmod 4)\} .
$$

However, we will see that the coefficients of $q g_{ \pm 1}\left(q^{2}\right)$ will always be equal up to absolute value, due to the following relations with $f(q)$ and $h(q)$ :

$$
\begin{aligned}
& \frac{1}{2}\left(g_{+1}(q)+g_{-1}(q)\right)=f(q), \\
& \frac{1}{2}\left(g_{+1}(q)-g_{-1}(q)\right)=q^{-\frac{1}{2}} h(q) .
\end{aligned}
$$

Taking $q \rightarrow q^{2}$ and adding the two above equations relates the coefficients of $g_{1}(q)$ to the arithmetic of $O_{K}$ by

$$
q g_{+1}\left(q^{2}\right)=q f\left(q^{2}\right)+h\left(q^{2}\right)=\sum_{\mathbf{a} \subset O_{K}}\left[\left(\frac{-4}{\mathcal{N}(\mathbf{a})}\right)-\frac{1+(-1)^{\mathcal{N}(\mathbf{a})}}{2} i^{\mathcal{N}(\mathbf{a})}\right] q^{\mathcal{N}(\mathbf{a})},
$$

which follows from equations (1.3) and (1.4). Here we have used the fact that there is a unique ideal of $O_{K}$ of norm 2, so $h\left(q^{2}\right)$ is precisely the generating function for the elements of norm $2 n$, weighted by $-i^{2 n}$. Subtracting the two equations yields

$$
q g_{-1}\left(q^{2}\right)=q f\left(q^{2}\right)-h\left(q^{2}\right)=\sum_{\mathbf{a} \subset O_{K}}\left[\left(\frac{-4}{\mathcal{N}(\mathbf{a})}\right)+\frac{1+(-1)^{\mathcal{N}(\mathbf{a})}}{2} i^{\mathcal{N}(\mathbf{a})}\right] q^{\mathcal{N}(\mathbf{a})} .
$$

Remark. Since $\left(\frac{-4}{2 n}\right)=0$ and $1+(-1)^{2 n+1}=0$, it follows that the absolute value of the $n$-th Fourier coefficient of both $q g_{+1}\left(q^{2}\right)$ and $q g_{-1}\left(q^{2}\right)$ equals the number of ideals of $O_{K}$ of norm $n$. Hence, although the coefficients of these series are not multiplicative, they are multiplicative up to \pm 1 .

We next show a sum of the tail-identity involving the functions $g_{z}$. For this, we let

$$
\mathcal{D}_{z}(q):=\sum_{n \geq 1} \frac{z^{-1} q^{n+\frac{1}{2}}}{1-z^{-1} q^{n+\frac{1}{2}}}-\sum_{n \geq 0} \frac{z q^{n-\frac{1}{2}}}{1-z q^{n-\frac{1}{2}}} .
$$

Theorem 2.1. We have

$$
\sum_{n \geq 0}\left(\frac{\left(z q^{\frac{1}{2}}\right)_{\infty}}{\left(-z q^{\frac{1}{2}}\right)_{\infty}}-\frac{\left(z q^{\frac{1}{2}}\right)_{n}}{\left(-z q^{\frac{1}{2}}\right)_{n+1}}\right)=\frac{\left(z q^{\frac{1}{2}}\right)_{\infty}}{\left(-z q^{\frac{1}{2}}\right)_{\infty}}\left(\mathcal{D}_{z}(q)-1+2 D_{2}(q)\right)-z^{-1} q^{\frac{1}{2}} g_{z}(q)
$$

This immediately implies the following corollary.

Corollary 2.2. The following equations hold

$$
\begin{aligned}
\sum_{n \geq 0}\left(\frac{\left(q^{\frac{1}{2}}\right)_{\infty}}{\left(-q^{\frac{1}{2}}\right)_{\infty}}-\frac{\left(q^{\frac{1}{2}}\right)_{n}}{\left(-q^{\frac{1}{2}}\right)_{n+1}}\right) & =2 \frac{\left(q^{\frac{1}{2}}\right)_{\infty}}{\left(-q^{\frac{1}{2}}\right)_{\infty}} D_{2}(q)-q^{\frac{1}{2}} g_{1}(q), \\
\sum_{n \geq 0}\left(\frac{\left(-q^{\frac{1}{2}}\right)_{\infty}}{\left(q^{\frac{1}{2}}\right)_{\infty}}-\frac{\left(-q^{\frac{1}{2}}\right)_{n}}{\left(q^{\frac{1}{2}}\right)_{n+1}}\right) & =2 \frac{\left(-q^{\frac{1}{2}}\right)_{\infty}}{\left(q^{\frac{1}{2}}\right)_{\infty}} D_{2}(q)+q^{\frac{1}{2}} g_{-1}(q) .
\end{aligned}
$$

Proof. ¿From Theorem 1 of [3] with $a=-z q^{\frac{1}{2}}$ and $t=z q^{-\frac{1}{2}}$, one may easily deduce that

$$
\sum_{n \geq 1}\left(\frac{\left(z q^{-\frac{1}{2}}\right)_{\infty}}{\left(-z q^{\frac{1}{2}}\right)_{\infty}}-\frac{\left(z q^{-\frac{1}{2}}\right)_{n}}{\left(-z q^{\frac{1}{2}}\right)_{n}}\right)=\frac{\left(z q^{-\frac{1}{2}}\right)_{\infty}}{\left(-z q^{\frac{1}{2}}\right)_{\infty}}\left(\mathcal{D}_{z}(q)-1+2 D_{2}(q)\right)+1+\sum_{n \geq 1} \frac{\left(-z^{-1} q^{\frac{1}{2}}\right)_{n}}{\left(z^{-1} q^{\frac{3}{2}}\right)_{n}}(-1)^{n} q^{n} .
$$


Dividing both sides by $1-z q^{-\frac{1}{2}}=-z q^{-\frac{1}{2}}\left(1-z^{-1} q^{\frac{1}{2}}\right)$ and shifting the sum of tails gives

$\sum_{n \geq 0}\left(\frac{\left(z q^{\frac{1}{2}}\right)_{\infty}}{\left(-z q^{\frac{1}{2}}\right)_{\infty}}-\frac{\left(z q^{\frac{1}{2}}\right)_{n}}{\left(-z q^{\frac{1}{2}}\right)_{n+1}}\right)=\frac{\left(z q^{\frac{1}{2}}\right)_{\infty}}{\left(-z q^{\frac{1}{2}}\right)_{\infty}}\left(\mathcal{D}_{z}(q)-1+2 D_{2}(q)\right)-z^{-1} q^{\frac{1}{2}} \sum_{n \geq 0} \frac{\left(-z^{-1} q^{\frac{1}{2}}\right)_{n}}{\left(z^{-1} q^{\frac{1}{2}}\right)_{n+1}}(-1)^{n} q^{n}$.

Using Heine's second transformation (cf. [1, p. 11, (2.15)]) with $a=b=q, c=-q^{2}$, and $z=z^{-1} q^{\frac{1}{2}}$ gives that

$$
g_{z}(q)=\sum_{n \geq 0} \frac{\left(-z^{-1} q^{\frac{1}{2}}\right)_{n}}{\left(z^{-1} q^{\frac{1}{2}}\right)_{n+1}}(-1)^{n} q^{n}
$$

This gives the claim.

\section{Proof of the Main Result}

In this section, we will use Theorem 2.2 to obtain the desired sum of tails results for $f(q)$ and $h(q)$. Since the proof of both identities are quite similar, we only prove the first here. We first define

$$
F(q):=\frac{\left(q^{\frac{1}{2}}\right)_{\infty}}{\left(-q^{\frac{1}{2}}\right)_{\infty}}+\frac{\left(-q^{\frac{1}{2}}\right)_{\infty}}{\left(q^{\frac{1}{2}}\right)_{\infty}}
$$

and its "finite companion"

$$
F_{n}(q):=\frac{\left(q^{\frac{1}{2}}\right)_{n}}{\left(-q^{\frac{1}{2}}\right)_{n+1}}+\frac{\left(-q^{\frac{1}{2}}\right)_{n}}{\left(q^{\frac{1}{2}}\right)_{n+1}} .
$$

Specializing equation (2.3) once with $z=1$ and once $z=-1$ and then summing gives

$$
\sum_{n \geq 0}\left(F(q)-F_{n}(q)\right)=2 F(q) D_{2}(q)-2 h(q) .
$$

Thus it remains to compute $F(q)$ and $F_{n}(q)$. Using the Jacobi triple product identity (cf. [5, p. 17]) one obtains

$$
\begin{aligned}
F\left(q^{2}\right) & =\frac{1}{\left(q^{2} ; q^{4}\right)_{\infty}\left(q^{2} ; q^{2}\right)_{\infty}}\left(\frac{(q)_{\infty}^{2}}{\left(q^{2} ; q^{2}\right)_{\infty}}+\frac{\left(q^{2} ; q^{2}\right)_{\infty}^{5}}{\left(q^{4} ; q^{4}\right)^{2}(q)_{\infty}^{2}}\right) \\
& =\frac{1}{\left(q^{2} ; q^{4}\right)_{\infty}\left(q^{2} ; q^{2}\right)_{\infty}}\left(\sum_{n \in \mathbb{Z}}(-1)^{n} q^{n^{2}}+\sum_{n \in \mathbb{Z}} q^{n^{2}}\right) .
\end{aligned}
$$

Thus

$$
F(q)=\frac{2}{\left(q ; q^{2}\right)_{\infty}(q)_{\infty}} \theta_{0}(q)
$$

To compute $F_{n}(q)$ we first rewrite

$$
F_{n}(q)=\frac{1}{\left(q ; q^{2}\right)_{n+1}}\left(\left(q^{\frac{1}{2}}\right)_{n}^{2}\left(1-q^{n+\frac{1}{2}}\right)+\left(-q^{\frac{1}{2}}\right)_{n}^{2}\left(1+q^{n+\frac{1}{2}}\right)\right)
$$


and then use McMahon's finite version of the Jacobi triple product identity [6, vol. 2, Section $323]$ with $x=q^{-\frac{1}{2}}$ for the first summand and $x=-q^{-\frac{1}{2}}$ for the second summand. This gives

$$
\begin{aligned}
F_{n}(q) & =\frac{1}{\left(q ; q^{2}\right)_{n+1}}\left(\left(1-q^{n+\frac{1}{2}}\right) \sum_{j \in \mathbb{Z}}\left[\begin{array}{c}
2 n \\
n+j
\end{array}\right](-1)^{j} q^{\frac{j^{2}}{2}}+\left(1+q^{n+\frac{1}{2}}\right) \sum_{j \in \mathbb{Z}}\left[\begin{array}{c}
2 n \\
n+j
\end{array}\right] q^{\frac{j^{2}}{2}}\right) \\
& =\frac{2}{\left(q ; q^{2}\right)_{n+1}}\left(\sum_{j \in \mathbb{Z}}\left[\begin{array}{c}
2 n \\
n+2 j
\end{array}\right] q^{2 j^{2}}+q^{n+\frac{1}{2}} \sum_{j \in \mathbb{Z}}\left[\begin{array}{c}
2 n \\
n+2 j+1
\end{array}\right] q^{2 j^{2}+2 j+\frac{1}{2}}\right) \\
& =\frac{2}{\left(q ; q^{2}\right)_{n+1}} \sum_{j \in \mathbb{Z}} \frac{(q)_{2 n}}{(q)_{n+2 j+1}(q)_{n-2 j}} q^{2 j^{2}}\left(1-q^{n+2 j+1}+q^{n+2 j+1}\left(1-q^{n-2 j}\right)\right) \\
& =\frac{2}{\left(q ; q^{2}\right)_{n+1}} \sum_{j \in \mathbb{Z}}\left[\begin{array}{c}
2 n+1 \\
n-2 j
\end{array}\right] q^{2 j^{2}}=\frac{2}{\left(q ; q^{2}\right)_{n+1}(q)_{n}} \theta_{0, n}(\tau) .
\end{aligned}
$$

There is additionally another sum of tails identity related to $g_{z}(q)$. In this case $g_{z}(q)$ does not play the role of the "error series" but rather as part of the "divisor function".

Theorem 3.1. We have the sum of tails identity

$$
\sum_{n \geq 0}\left(\frac{(-q)_{\infty}}{(q)_{\infty}}-\frac{(-q)_{n}}{(q)_{n}\left(1-q^{2 n+1}\right)}\right)=\frac{(-q)_{\infty}}{(q)_{\infty}}\left(2 D_{2}(q)-h(q)\right) .
$$

Proof. ¿From Theorem 2 of [3] with $a=-q, b=z^{-1} q^{\frac{1}{2}}$, and $c=z^{-1} q^{\frac{3}{2}}$, one obtains

$$
\begin{aligned}
\sum_{n \geq 0}\left(\frac{(-q)_{\infty}}{(q)_{\infty}}-\frac{(-q)_{n}}{(q)_{n}\left(1-z^{-1} q^{n+\frac{1}{2}}\right)}\right) & =\frac{(-q)_{\infty}}{(q)_{\infty}}\left(2 \sum_{n \geq 1} \frac{q^{n}}{1-q^{2 n}}-\sum_{n \geq 1} \frac{(q)_{n}}{(-q)_{n}\left(1-q^{n}\right)} z^{-n} q^{\frac{n}{2}}\right) \\
& =\frac{(-q)_{\infty}}{(q)_{\infty}}\left(2 D_{2}(q)-z^{-1} q^{\frac{1}{2}} g_{z}(q)\right) .
\end{aligned}
$$

The result then follows after summing the terms with $z=1$ and $z=-1$ and dividing by 2 .

In addition to these sum of tails identities, the auxilliary function $g_{z}(q)$ also allows us to deduce new combinatorial interpretations for $f(q)$ and $h(q)$ in terms of overpartitions. Using Heine's first transformation (cf. [1, p. 10, (2.11)]) with $a=b=q, c=-q^{2}$, and $z=z^{-1} q^{\frac{1}{2}}$ gives

$$
g_{z}(q)=\frac{(q)_{\infty}}{(-q)_{\infty}} \sum_{n \geq 0} \frac{(-q)_{n}}{(q)_{n}\left(1-z^{-1} q^{n+\frac{1}{2}}\right)} q^{n} .
$$

After pulling the infinite product inside the sum, this immediately gives

$$
f(q)=\sum_{n \geq 0} \frac{\left(q^{n+1}\right)_{n}\left(q^{2 n+2}\right)_{\infty}}{\left(-q^{n+1}\right)_{\infty}} q^{n}, \quad h(q)=\sum_{n \geq 0} \frac{\left(q^{n+1}\right)_{n}\left(q^{2 n+2}\right)_{\infty}}{\left(-q^{n+1}\right)_{\infty}} q^{2 n+1} .
$$

To describe the new combinatorial interpretation for the coefficients of $f$ and $h$, let $n=s(\Lambda)$ denote the smallest part of the overpartition $\Lambda$. Then $-f(q)$ is the generating function for overpartitions where $s(\Lambda)$ is overlined and occurs exactly once and $2 s(\Lambda)+1$ cannot be overlined, weighted by $(-1)^{M(\Lambda)}$. 
NEW IDENTITIES INVOLVING SUMS OF THE TAILS RELATED TO REAL QUADRATIC FIELDS

Similarly, writing $2 n+1=n+(n+1)$, we see that $h(q)$ is the generating function for overpartitions for which $s(\Lambda)$ is overlined and occurs precisely once, a non-overlined part of size $s(\Lambda)+1$ must occur, and $2 s(\Lambda)+1$ cannot be overlined, again weighted by $(-1)^{M(\Lambda)}$.

\section{VAlues of ZEta FUnCtions AT NeGative INTEGERS}

We will use Theorem 1.1 to establish Theorem 1.2 here.

Proof of Theorem 1.2. This proof will follow analogously to that of Zagier [8, Theorem 3] so we will give a brief argument for (1.7) and leave the details of (1.8) to the reader. We first note that

$$
\frac{\theta_{0}(q)}{(q)_{\infty}\left(q ; q^{2}\right)_{\infty}}=\frac{(-q)_{\infty}^{2}\left(-q^{2} ; q^{2}\right)_{\infty}^{3}}{\left(-q^{4} ; q^{4}\right)_{\infty}^{2}}
$$

and hence vanishes to infinite order as $q \rightarrow-1$. We next replace $q$ by $-e^{-t}$ and define $a(n)$ and $b(n)$ by the asymptotic expansions as $t \rightarrow 0$ given by

$$
\begin{aligned}
-\sum_{n \geq 0} \frac{\theta_{0, n}\left(-e^{-t}\right)}{\left(-e^{-t} ;-e^{-t}\right)_{n}\left(-e^{-t} ; e^{-2 t}\right)_{n+1}} & =\sum_{n \geq 0} a(n) t^{n}, \\
-h\left(-e^{-t}\right):=\sum_{\mathbf{a} \subset O_{K}} e^{-t \mathcal{N}(\mathbf{a})} & \sim \sum_{n \geq 0} b(n) t^{n} .
\end{aligned}
$$

By Theorem 1.1 we have $a(n)=b(n)$ for every $n \geq 0$. We then integrate to obtain

$$
-\int_{0}^{\infty} h\left(-e^{-t}\right) t^{s-1} d t=\sum_{\mathbf{a} \subset O_{K}} \int_{0}^{\infty} e^{-t \mathcal{N}(\mathbf{a})} t^{s-1} d t=\Gamma(s) \sum_{\mathbf{a} \subset O_{K}} \mathcal{N}(\mathbf{a})^{-s}=\Gamma(s) \zeta_{K}(s) .
$$

The residue at $s=-n$ is hence given by $\frac{(-1)^{n}}{n !} \zeta_{K}(-n)$. However, for any $N$ fixed we have

$$
-\int_{0}^{\infty} h\left(-e^{-t}\right) t^{s-1} d t=\int_{0}^{\infty}\left(\sum_{0 \leq n<N} b(n) t^{n}+O\left(t^{N}\right)\right) t^{s-1} d t=\sum_{0 \leq n<N} \frac{b(n)}{s+n}+H(s),
$$

where $H(s)$ is holomorphic for $\operatorname{Re}(s)>-N$, so that for $n<N$ the residue at $s=-n$ is $b(n)$.

\section{REFERENCES}

[1] G. Andrews, q-Series: Their Development and Application in Analysis, Number Theory, Combinatorics, Physics, and Computer Algebra, CBMS Regional Conference Series in Mathematics, no. 66, Amer. Math. Soc. , Providence, RI, pp. 130, 1986.

[2] G. Andrews, F. Dyson, and D. Hickerson, Partitions and indefinite quadratic forms, Invent. Math. 91 (1988), pages 391-407.

[3] G. Andrews, J. Jimenez-Urroz, K. Ono, q-series identities and values of certain L-functions, Duke Math. J. 108 (2001), pages 395-419.

[4] K. Bringmann, B. Kane, Multiplicative q-hypergeometric series arising from real quadratic fields, preprint, arxiv:0812.4397.

[5] K. Ono, Web of modularity: Arithmetic of the coefficients of modular forms and q-series, CBMS Regional Conference Series in Mathematics, no. 102, Amer. Math. Soc., Providence, RI, 2003.

[6] P. A. MacMahon, Combinatory Analysis vol. I, II. Cambridge Univ. Press, 1915. Reprinted by Chelsea, New York, 1960.

[7] S. Ramanujan, The lost notebook and other unpublished papers, Narosa, New Delhi, 1988.

[8] D. Zagier, Vassiliev invariants and a strange identity related to the Dedekind eta-function, Topology 40 (2001), pages 945-960. 
Mathematical Institute, University of Cologne, Weyertal 86-90, 50931 Cologne, Germany E-mail address: kbringma@math.uni-koeln.de

Math Department, Radboud University, Postbus 9010, 6500 GL, Nijmegen, Netherlands E-mail address: bkane@science.ru.nl 